\title{
Spatial analysis of aquifer response times for radial flow processes: Nondimensional analysis and laboratory-scale tests
}

Farhad Jazaei ${ }^{1},{ }^{*}$ Matthew J Simpson ${ }^{2}$ and T Prabhakar Clement ${ }^{1}$

1 Department of Civil Engineering, Auburn University, Auburn AL, 36849. USA.

2 School of Mathematical Sciences, Queensland University of Technology. GPO Box 2434, Brisbane, Queensland 4001, Australia. 


\begin{abstract}
A fundamental concept in groundwater hydrology is the notion of steady state, or equilibrium conditions. When a system at some initial steady state condition is perturbed by pumping, a transient cone of depression will develop and the system will approach a new steady state condition. Understanding the time scale required for the transient process to occur is of practical interest since it would help practitioners decide whether to use a steady state model or a more complicated transient model. Standard approaches to estimate the response time use simple scaling relationships which neglect spatial variations. Alternatively, others define the response time to be the amount of time taken for the difference between the transient and steady state solutions to fall below some arbitrary tolerance level. Here, we present a novel approach and use the concept of mean action time to predict aquifer response time scales in a two-dimensional radial geometry for pumping, injection and recovery processes. Our approach leads to relatively simple closed form expressions that explicitly show how the time scale depends on the hydraulic parameters and position. Furthermore, our dimensionless framework allows us to predict the response time scales for a range of applications including small scale laboratory problems and large scale field problems. Our analysis shows that the response time scales vary spatially, but are equivalent for pumping, injection and associated recovery processes. Furthermore, the time scale is independent of the pumping or injection flow rate. We test these predictions in a laboratory scale aquifer and find that our physical measurements corroborate the theoretical predictions.
\end{abstract}

Key words: Pumping, Injection, Recovery, Response time scale, Mean action time

\footnotetext{
* Corresponding author

Email address: matthew.simpson@qut.edu.au
} 


\section{$1 \quad 1$ Introduction}

2 Population growth and associated industrial and agricultural activities can

3 have considerable impact on groundwater resources. Since groundwater plays

4 a significant role in our social and economic wellbeing, understanding ground-

5 water responses to natural and anthropogenic changes is important. Several

6 studies have examined various properties of groundwater flow processes using

7 different tools including numerical or analytical models, field investigations

and laboratory experiments (e.g. Theis, 1935; Freeze and Witherspoon, 1966;

9 Bredehoeft et al., 1982; Hantush, 2005). Many of these studies have included

10 radial flow problems to investigate pumping, injection and recovery processes.

11 A common concept used in groundwater modeling is defining a steady state

12 (or equilibrium) flow condition. When a forcing condition on a system at equi-

13 librium is changed, the system will undergo a transient response to approach a

14 new equilibrium state. A point of interest is to understand the amount of time

15 taken for the system to reach steady state. Strictly speaking, from a mathe-

16 matical point of view, an infinite amount of time is required for the system

17 to asymptote to steady state conditions. However, this strict mathematical

18 definition is impractical because we can never wait for an infinite amount of

19 time. Therefore, we wish to estimate a "sufficiently long period" of time that

20 is required for the system to "effectively" reach steady state (Schwartz et al.,

21 2010). However, the concept of a "sufficiently long period" is subtle.

22 A change in flow conditions at a pumping or injection well will eventually 23 influence regions further away from the well, potentially over very large areas, 24 including distant boundary conditions. When the flow rate at a pumping or 25 injection well is altered, a transition pattern, often called a cone of depression, propagates through the aquifer with time. Understanding the amount of time

27 required for a transient system to effectively relax to equilibrium can help us

decide whether to use a steady state model or a more complicated transient 
model to describe the groundwater flow process (Simpson et al., 2013; Jazaei et al., 2014).

The concept of aquifer response time has been analyzed previously for various groundwater problems. Theis first considered the response of a groundwater system to pumping by solving a mathematical model describing the transient flow near a pumping well in an infinite aquifer (Theis, 1935). After this initial study, Theis then considered the factors controlling the response time (Theis, 1940). These factors include the aquifer transmissivity, $T$; the storage coefficient, $S$; and the length scale of the problem. Theis concluded that the rate at which the cone of depression spreads is proportional to $T$ and inversely proportional to $S$. Later, other researchers presented simpler scaling formulas to estimate the aquifer response time scale (e.g. Gelhar and Wilson, 1974; Townley, 1995; Erskine and Papaionnau, 1997; Manga, 1999; Haitjema, 2006). For example, Gelhar and Wilson (1974) suggest that the hydraulic response time is $t_{h}=n L^{2} / 3 T$, where $n$ is the average porosity and $L$ is the aquifer length. Such scaling formulas suggest a constant time scale for the entire system and do not provide any information about how the time scale depends on position. Other studies (e.g. Schwartz et al., 2010, Kooi and Groen, 2000; Rousseau-Gueutin et al., 2013) define the response time as the amount of time taken for the difference between the transient and steady state solutions to fall below some tolerance. For example Rousseau-Gueutin et al. (2013) define the aquifer response time to be the amount of time required for $95 \%$ of the transient head changes to have occurred. This definition does not lead to a simple closed form expression.

Recently, we presented a different framework to quantify the aquifer response time scale (Simpson et al., 2013; Jazaei et al., 2014). Our analysis provides explicit mathematical expressions showing how the response time scale depends on position, aquifer properties and boundary conditions. This approach does not require any predefined thresholds, and avoids the need for solving 
the transient flow problem. However, our previous analyses were limited to one-dimensional Cartesian problems in which flows where driven by a surface recharge conditions, or changes at the interface between the surface water and groundwater. In contrast, here we analyze the time scale of a two-dimensional radial system, in which the transition between different steady state conditions is driven by flow changes at the pumping or injecting well. Our analysis is relevant for both converging and diverging flows and we employ a nondimensional framework which leads to more elegant, generalized results, which can be used to explain the difference between smaller scale laboratory flow conditions and larger scale field conditions.

Our approach involves analyzing the first and second moments of the transition time distribution, which is similar to the way in which some previous studies have used temporal moment analysis to investigate spatial variations in hydraulic conductivity (Wei, 2005; Zhu and Yeh, 2006). We note, however, these previous studies were focusing on analyzing the hydraulic conductivity fields, and did not consider using moment analysis to derive expressions for the aquifer response time scales.

The objective of the present work is to develop a framework to quantify the spatial variations in response time scales under radial flow conditions. We investigate pumping, injection and recovery processes to understand how their response time scales depend on hydraulic and geometric properties of the aquifer. We employ two mathematical concepts, known as the mean action time (MAT) and the variance of action time (VAT) in this analysis. We employ a dimensionless framework that can be used to study both large scale field problems as well as small scale laboratory problems. Our theoretical predictions are tested using new datasets from laboratory scale experiments. 
In this section we first use a dimensional radial flow model to define a simpler and more general dimensionless model. Primed variables denote dimensional quantities and unprimed variables denote dimensionless quantities.

\subsection{Dimensional model}

Groundwater flow near a fully penetrating well of radius $r_{w}^{\prime}$ in a homogeneous confined aquifer can be analyzed using the following dimensional model (Bear, 1976),

$$
S \frac{\partial h^{\prime}\left(r^{\prime}, t^{\prime}\right)}{\partial t^{\prime}}=\frac{T^{\prime}}{r^{\prime}} \frac{\partial}{\partial r^{\prime}}\left[r^{\prime} \frac{\partial h^{\prime}\left(r^{\prime}, t^{\prime}\right)}{\partial r^{\prime}}\right], \quad r_{w}^{\prime}<r^{\prime}<R^{\prime},
$$

where $r^{\prime}[\mathrm{L}]$ is the radial distance from the center of the well, $t^{\prime}[\mathrm{T}]$ is time, $R^{\prime}[\mathrm{L}]$ is the radial distance between the center of the well and the boundary, $h^{\prime}\left(r^{\prime}, t^{\prime}\right)[\mathrm{L}]$ is the hydraulic head, $T^{\prime}\left[\mathrm{L}^{2} / \mathrm{T}\right]$ is the aquifer transmissivity and $S[-]$ is the aquifer storage coefficient. In this study we consider a Dirichlet boundary condition, $h^{\prime}\left(R^{\prime}, t^{\prime}\right)=h_{0}^{\prime}$.

For both pumping and injection processes we consider a spatially uniform initial condition, $h_{0}^{\prime}\left(r^{\prime}\right)=h_{0}^{\prime}$, and a constant flow rate denoted $Q^{\prime}>0$ for pumping and $Q^{\prime}<0$ for injection. For the radial problem the steady state solution is

$$
\lim _{t^{\prime} \rightarrow \infty} h^{\prime}\left(r^{\prime}, t^{\prime}\right)=h_{\infty}^{\prime}\left(r^{\prime}\right)=h_{0}^{\prime}+\frac{Q^{\prime}}{2 \pi T^{\prime}} \ln \left(\frac{r^{\prime}}{R^{\prime}}\right) .
$$

The transient solution of Eq. (1) can be written as an infinite series involving Bessel functions (Bear, 1976). We remark that the Theis solution is relevant only for aquifers of infinite extent and therefore does not apply to the finite problems considered here.

For the recovery process, we suppose that the flow rate at the well is stopped, giving $Q^{\prime}=0$. Furthermore, we assume that the initial condition corresponds 
and two constants that are given by:

$$
\alpha=\frac{r_{w}^{\prime}}{R^{\prime}}, \quad \beta=\frac{Q^{\prime}}{2 \pi T^{\prime} h_{0}^{\prime} \alpha}
$$

117 Physically, $\alpha$ is a positive constant representing the ratio of the well radius, $r_{w}^{\prime}$,

$$
2 \pi r_{w}^{\prime} T^{\prime} \frac{\partial h^{\prime}\left(r_{w}^{\prime}, t^{\prime}\right)}{\partial r^{\prime}}-Q^{\prime}=\pi r_{w}^{\prime}{ }_{2} \frac{\partial h^{\prime}\left(r_{w}^{\prime}, t^{\prime}\right)}{\partial t^{\prime}}
$$

11 The boundary condition at $r^{\prime}=R^{\prime}$ is given by

$$
h^{\prime}\left(R^{\prime}, t^{\prime}\right)=h_{0}^{\prime}
$$

\subsection{Dimensionless model}

$$
r^{*}=R^{\prime}, \quad t^{*}=\frac{S R^{\prime 2}}{T^{\prime}}, \quad h^{*}=h_{0}^{\prime} .
$$

We then define the following three dimensionless variables,

$$
r=\frac{r^{\prime}}{r^{*}}, \quad t=\frac{t^{\prime}}{t^{*}}, \quad h=\frac{h^{\prime}}{h^{*}},
$$

18 to the length of the domain, $R^{\prime}$. For a small scale laboratory problem we have $\alpha \approx 0.01$ whereas in a field scale application $R^{\prime} \gg r_{w}^{\prime}$, so we are interested in the limiting condition, $\alpha \rightarrow 0$. Working in this dimensionless framework we can extend our analysis to both laboratory scale and field scale applications by simply varying $\alpha$. 
Substituting dimensionless variables and constants into Eqs. (1), (3) and (4), we obtain a simpler dimensionless model,

$$
\begin{array}{ll}
\frac{\partial h(r, t)}{\partial t}=\frac{1}{r} \frac{\partial}{\partial r}\left[r \frac{\partial h(r, t)}{\partial r}\right], & \alpha<r<1, \\
\frac{\partial h(\alpha, t)}{\partial r}-\beta=\frac{\alpha}{2 S} \frac{\partial h(\alpha, t)}{\partial t}, & h(1, t)=1 .
\end{array}
$$

The dimensionless initial condition for pumping and injection processes is $h_{0}(r)=1$. The associated steady state solution is $h_{\infty}(r)=\alpha \beta \ln (r)+1$. We assume that the initial condition for the recovery process is equivalent to the steady state condition for the pumping or injection process. The dimensionless steady solution of the recovery process is $h_{\infty}(r)=1$.

\section{Mean action time for a radial system}

In this section we derive expressions for the MAT of the system using the dimensionless model. This approach leads to an expression for the dimensionless mean time scale, $M(r)$, which can be rescaled to give a dimensional time scale by multiplying by $t^{*}$. We begin by defining two mathematical quantities (McNabb and Wake, 1991; McNabb, 1993),

$$
\begin{aligned}
& F(t \mid r)=1-\frac{h(r, t)-h_{\infty}(r)}{h_{0}(r)-h_{\infty}(r)}, \quad t \geq 0, \\
& f(t \mid r)=\frac{\mathrm{d} F(t \mid r)}{\mathrm{d} t}=-\frac{\partial}{\partial t}\left[\frac{h(r, t)-h_{\infty}(r)}{h_{0}(r)-h_{\infty}(r)}\right], \quad t \geq 0 .
\end{aligned}
$$

To apply these quantities to a particular problem we require $h_{0}(r) \neq h_{\infty}(r)$ to ensure a transition occurs. Hence, for our problem, Eqs. (10) and (11) are indeterminate at $r=1$ and we will explain how to deal with this later.

For all pumping and injection processes, $h(r, t)$ changes monotonically from $h_{0}(r)$ to $h_{\infty}(r)$. Therefore, $F(t \mid r)$ is a monotonically increasing function with an initial value of $F(0 \mid r)=0$, and approaches unity as $t \rightarrow \infty$. We interpret $F(t \mid r)$ as a cumulative distribution function (CDF). $F(t \mid r)$ quantifies the 
amount of action completed at a position $r$, after time $t$. Note that here we treat $t$ as the independent variable and $r$ as a parameter. At $t=0$, the transition is yet to begin, hence $F(0 \mid r)=0$. As the process proceeds, the value of $F(t \mid r)$ increases. For example, we interpret $F(\delta \mid r)=0.5$ as indicating that $50 \%$ of the transient process is completed after $t=\delta$ at position $r$.

By definition, the first derivative of $F(t \mid r)$ with respect to $t$, given by Eq. (11), is the probability density function (PDF) (Ellery et al., 2012a,b). Mathematically, $f(t \mid r)$ is proportional to the time derivative of $h(r, t)$ at each position $r$. Therefore, $f(t \mid r) \rightarrow 0$ as $t \rightarrow \infty$, and the system reaches steady state.

The MAT is the first moment of $f(t \mid r)$ (McNabb and Wake, 1991; McNabb, 1993),

$$
M(r)=\int_{0}^{\infty} t f(t \mid r) \mathrm{d} t
$$

Since we know that $h(r, t)$ decays to $h_{\infty}(r)$ exponentially fast as $t \rightarrow \infty$ (Crank, 1975), applying integration by parts to Eq. (12) leads to

$$
M(r) g(r)=\int_{0}^{\infty}\left(h_{\infty}(r)-h(r, t)\right) \mathrm{d} t
$$

where, $g(r)=h_{\infty}(r)-h_{0}(r)$. Differentiating Eq. (13) twice with respect to $r$ gives,

$$
\begin{aligned}
& \frac{\mathrm{d}[M(r) g(r)]}{\mathrm{d} r}=\int_{0}^{\infty}\left(\frac{\mathrm{d} h_{\infty}(r)}{\mathrm{d} r}-\frac{\partial h(r, t)}{\partial r}\right) \mathrm{d} t, \\
& \frac{\mathrm{d}^{2}[M(r) g(r)]}{\mathrm{d} r^{2}}=\int_{0}^{\infty}\left(\frac{\mathrm{d}^{2} h_{\infty}(r)}{\mathrm{d} r^{2}}-\frac{\partial^{2} h(r, t)}{\partial r^{2}}\right) \mathrm{d} t .
\end{aligned}
$$

Multiplying Eq. (14) by 1/r and combining the resulting expression with Eqs. (8) and (15), we obtain a boundary value problem for the MAT:

$$
\frac{\mathrm{d}^{2} M(r)}{\mathrm{d} r^{2}}+\frac{\mathrm{d} M(r)}{\mathrm{d} r}\left[\frac{1}{r}+\frac{2}{g(r)} \frac{\mathrm{d} g(r)}{\mathrm{d} r}\right]+\frac{M(r)}{g(r)}\left[\frac{1}{r} \frac{\mathrm{d} g(r)}{\mathrm{d} r}+\frac{\mathrm{d}^{2} g(r)}{\mathrm{d} r^{2}}\right]=-1
$$

Eq. (16) is valid for an arbitrary initial condition, $h_{0}(r)$. However, in this study 1 we consider a spatially uniform initial condition, $h_{0}(r)=1$, which allows us 
to simplify Eq. (16) to

$$
\frac{\mathrm{d}^{2} M(r)}{\mathrm{d} r^{2}}+\left[\frac{2+\ln (r)}{r \ln (r)}\right] \frac{\mathrm{d} M(r)}{\mathrm{d} r}=-1 .
$$

To solve Eq. (17) we require boundary conditions at $r=\alpha$ and $r=1$. At $r=\alpha$, we use Eqs. (9) and (14) to define a Robin condition given by

$$
\frac{\mathrm{d} M(\alpha)}{\mathrm{d} r}+\frac{M(\alpha)}{\alpha \ln (\alpha)}=-\frac{\alpha}{2 S} .
$$

Since $h(r, t)$ decays to $h_{\infty}(r)$ exponentially fast as $t \rightarrow \infty, f(t \mid r)$ must also decay to zero exponentially fast as $t \rightarrow \infty$. Therefore, $M(r)$, defined by Eq. (12), must be finite at all locations. The coefficient of $\mathrm{d} M(r) / \mathrm{d} r$ in Eq. (17) is infinite when $r \rightarrow 1$, implying that $M(r)$ is not well defined. To ensure that $M(r)$ is finite, we require

$$
\frac{\mathrm{d} M(1)}{\mathrm{d} r}=0
$$

Solving Eq. (17) with these boundary conditions gives us

$$
M(r)=\underbrace{\frac{r^{2}(1-\ln (r))-1}{4 \ln (r)}}_{\text {geometry term }}+\underbrace{C_{1}}_{\text {hydraulic term }},
$$

where the constant $C_{1}$ is given by

$$
C_{1}=\frac{\alpha^{2}(2 \ln (\alpha)-1)}{4}-\frac{\alpha^{2} \ln (\alpha)}{2 S} .
$$

\footnotetext{
Our solution for $M(r)$ represents the dimensionless mean time scale for the radial flow process at each position, $r$. The expression for $M(r)$ is the sum of two distinct 'geometry' and 'hydraulic' terms. The geometry term describes the spatial variation of the mean time scale. Intuitively, we expect that points closer to the well will respond faster than points further away from the well and the geometry term reflects this. The constant hydraulic term is independent of position and describes the storage effects associated with both the aquifer and the well.
}

The mathematical expression for $M(r)$, given by Eq. (20), is independent of 
$Q^{\prime}$, indicating that the mean time scale is equivalent for pumping and injection processes. Furthermore, the boundary value problem is invariant if we switch the roles of $h_{\infty}(r)$ and $h_{0}(r)$, which is the same as replacing $g(r)$ with $-g(r)$ throughout. Therefore, not only $M(r)$ is equivalent for pumping and injection processes, but it is also equivalent for the associated recovery process (Simpson et al. 2013).

It is interesting to remark that we obtained our expression for $M(r)$ without solving the governing flow equation for $h(r, t)$. The expression for $M(r)$ is relatively straightforward and does not depend on any arbitrary thresholds. Since we did not make any subjective choice of thresholds, and we only used standard definitions of the mean of a PDF, our expression for $M(r)$ is a fundamental, objectively defined quantity that shows how the mean time scale depends on relevant hydraulic and geometric properties of the problem. Plots in Fig. 1(a) illustrate how $M(r)$ varies with position for two different problems chosen to illustrate the differences between a field scale application, relevant for $\alpha \rightarrow 0$, and a laboratory scale application, with $\alpha>0$. Both plots show that the mean time scale increases with $r$, as expected.

It is useful to note that Eq. (20) can be simplified when $\alpha \rightarrow 0$, for field scale applications, by evaluating the expression for $C_{1}$ in the limit as $\alpha \rightarrow 0$, giving $C_{1}=0$. Therefore, for a field scale problem, the MAT is totally independent of well storage effects because $C_{1}=0$. Therefore, for a field scale problem, $M(r)$ depends only on the geometry. Conversely, for smaller laboratory scale problems, well storage effects play a role and our general expression for $M(r)$ allows us to quantify the relative importance of these effects for problems at different scales simply by varying $\alpha$.

Fig. 1 about here ... 


\section{Variance of action time for a radial system}

The VAT, $V(r)$, is the second moment of $f(t \mid r)$ (Simpson et al., 2013; Jazaei et al., 2014). Once we have evaluated $V(r)$, we can compute $\sqrt{V(r)}$, which is a measure of the spread of $f(t \mid r)$ about $M(r)$. An estimate of the time needed to reach steady state, after accounting for both the mean and variance of the PDF, can be written as,

$$
\tau(r)=M(r)+m \sqrt{V(r)}
$$

where $m$ is a positive integer. In this study, we begin by making a simplest possible choice by setting $m=1$, and we will discuss this choice later when we compare our theoretical predictions with our measurements from a physical model. This implies that our definition of time $\tau(r)$ is subjective since it depends on the choice of $m$. In contrast our definition of time scale $M(r)$ is completely objective.

The VAT is defined as (Simpson et al., 2013; Jazaei et al., 2014)

$$
V(r)=\int_{0}^{\infty}(t-M(r))^{2} f(t \mid r) \mathrm{d} t
$$

Using Eq. (12), together with the fact that $h(r, t)$ decays to $h_{\infty}(r)$ exponentially fast as $t \rightarrow \infty$, we rewrite Eq. (23) as,

$$
\phi(r)=2 \int_{0}^{\infty} t\left(h_{\infty}(r)-h(r, t)\right) \mathrm{d} t
$$

where,

$$
\phi(r)=g(r)\left[V(r)+M(r)^{2}\right] .
$$

23 By combining the first and the second derivatives of $\phi(r)$ with Eqs. (8) and 24 (12), we obtain a boundary value problem for $\phi(r)$,

$$
\frac{\mathrm{d}^{2} \phi(r)}{\mathrm{d} r^{2}}+\frac{1}{r} \frac{\mathrm{d} \phi(r)}{\mathrm{d} r}=-2 g(r) M(r) .
$$

225 To obtain the boundary conditions required to solve Eq. (26) we evaluate the 
first derivative of $\phi(r)$ at $r=\alpha$, and using Eq. (9), we obtain a Neumann boundary condition given by

$$
\frac{\mathrm{d} \phi(\alpha)}{\mathrm{d} r}=-\frac{\alpha M(\alpha) g(\alpha)}{S}
$$

Since $h(r, t)$ decays to $h_{\infty}(r)$ exponentially fast as $t \rightarrow \infty$, Eq. (23), guarantees that $V(r)$ is finite at all locations. Since $g(1)=0$, and $V(r)+M(r)^{2}$ is finite at all locations, the relevant boundary condition at $r=1$ is:

$$
\phi(1)=0
$$

Solving Eq. (26) with these boundary conditions gives,

$$
V(r)=\frac{1}{64}\left[\frac{128 C_{2}}{S}-2\left(r^{4}+32 C_{1}^{2}\right)+\frac{\left(5 r^{4}+128 C_{3}+32 C_{1}\right)}{\ln (r)}-\frac{4\left(r^{2}-1\right)^{2}}{\ln ^{2}(r)}\right],
$$

where $C_{1}$ is the hydraulic factor defined in Eq. (21) and,

$$
C_{2}=\frac{\alpha^{2}}{64}\left[4 \ln (\alpha)\left(8 S C_{1}-S \alpha^{2}+2 \alpha^{2}-8 C_{1}\right)+S\left(5 \alpha^{2}-16 C_{1}-8\right)-8 \alpha^{2}+8\right],
$$

$$
C_{3}=-\frac{5+32 C_{1}}{128}
$$

It is interesting to note that $V(r)$ is also independent of $Q^{\prime}$, and this shows that the width of $f(t \mid r)$ is same for pumping, injection and recovery processes. Now that we have closed form solutions for $M(r)$ and $V(r)$, we can calculate $\tau(r)=M(r)+m \sqrt{V(r)}$. Similar to our expression for $M(r)$, the expression for $V(r)$ can be simplified considerably for field scale problems by considering the limit that $\alpha \rightarrow 0$, giving $C_{1}=C_{2}=0$. However, unlike the expression for $M(r)$, the expression for $V(r)$ is more complicated and it is not obvious how to give these terms a meaningful interpretation like we did for $M(r)$.

Plots in Fig. 1(b)-(c) illustrate how $\sqrt{V(r)}$ and $\tau(r)$ (with $m=1$ ) varies with position for two different problems chosen to illustrate the differences between 
a field scale application and a laboratory scale application. Comparing the results for $M(r)$ and $\sqrt{V(r)}$ we see that the spatial variations in the mean is more pronounced than the spatial variations in the standard deviation.

\section{Laboratory experiments}

We now apply our method of calculating $M(r), V(r)$ and $\tau(r)$ to a set of laboratory scale pumping, injection and recovery experiments. The experiments involve a cylindrical confined flow system with a constant head boundary condition. Fig. 2 shows horizontal and vertical cross sections through the flow tank. The tank has two distinct chambers separated by a fine screen. The lower layer was wet packed with homogeneously mixed silty sand to form a circular confined aquifer of thickness $B^{\prime}=10 \mathrm{~cm}$, and a radius of $R^{\prime}=25$ $\mathrm{cm}$. The upper layer contained dry coarse sand and gravel material, primarily to support the confining plastic liner against the uplifting hydraulic pressure generated within the aquifer. A fully penetrating well of radius of $0.7 \mathrm{~cm}$ was located at the center of the aquifer. A thin tube with the radius of $0.3 \mathrm{~cm}$ connected to a peristaltic pump (Masterflex: L/S-7523-80) was inserted into the well to deliver water into or out of the aquifer. The effective radius of the well is approximately $r_{w}^{\prime}=\sqrt{0.7^{2}-0.3^{2}} \approx 0.6 \mathrm{~cm}$. The well was only screened in the lower layer and the wall was sealed at the upper layer with a plastic liner. A layer of clay was used near to the wall to prevent water leaking from the aquifer to the upper layer. Two syphon-type tubes were connected to electronic manometers to monitor head changes at $r_{1}^{\prime}=10 \mathrm{~cm}$ and $r_{2}^{\prime}=20 \mathrm{~cm}$. Head changes were monitored using 1 second time intervals, at a resolution of $0.1 \mathrm{~mm}$. The ends of the syphon-type tubes were screened to prevent solid particles to enter the tubes. The confined system was monitored for three days and no leakage was detected through the confining layer. The hydraulic conductivity of the silty sand material was independently measured 
to be $K^{\prime}=0.0008 \mathrm{~cm} / \mathrm{sec}$ using a falling head permeameter. Using our estimates of $K^{\prime}$ and $B^{\prime}$, we calculate the transmissivity, $T^{\prime}=K^{\prime} B^{\prime}$. The value of the storage coefficient was estimated to be $S=0.014$ by fitting a transient head dataset collected at $r_{1}^{\prime}=10 \mathrm{~cm}$ under a constant injection rate of 30 $\mathrm{ml} / \mathrm{min}$.

Fig. 2 about here ...

\subsection{Analysis of transient head data}

We performed one pumping and two injection experiments. We refer to these experiments as Experiment-P, Experiment-I1 and Experiment-I2, respectively. We also consider three associated recovery experiments, namely ExperimentRP, Experiment-RI1 and Experiment-RI2. The flow rates for Experiment-P, Experiment-I1 and Experiment-I2 are: $Q_{P}^{\prime}=15 \mathrm{ml} / \mathrm{min}, Q_{I 1}^{\prime}=-30 \mathrm{ml} / \mathrm{min}$, and $Q_{I 2}^{\prime}=-40 \mathrm{ml} / \mathrm{min}$, respectively. The initial and boundary conditions for all experiments is $h_{0}^{\prime}\left(r^{\prime}\right)=h^{\prime}\left(R^{\prime}, t^{\prime}\right)=35 \mathrm{~cm}$. Using Eq. (5), the characteristic length, time, and head scales for our experiments are:

$$
r^{*}=25 \mathrm{~cm}, \quad t^{*}=18.23 \mathrm{~min}, \quad h^{*}=35 \mathrm{~cm} .
$$

These quantities allow us to define dimensionless $r, t$ and $h$ using Eq. (6). Transient head changes were measured at $r_{1}^{\prime}=10 \mathrm{~cm}$ and $r_{2}^{\prime}=20 \mathrm{~cm}$, or $r_{1}=0.4$ and $r_{1}=0.8$, respectively. For our experimental system we have $\alpha=0.024$.

We predict the theoretical $M(r)$ and $V(r)$ values for six radial flow processes at two different positions using Eqs. (20) and (29), respectively. We also measured the observed time scales, which we refer to as experimental time scales using our laboratory datasets. To calculate MAT and VAT from our laboratory datasets we used the monitored transient head data to construct $f(t \mid r)$. To 
achieve this, we use a central difference approximation to estimate $\partial h / \partial t$. After constructing $f(t \mid 0.4)$ and $f(t \mid 0.8)$, we calculated the experimental $M(r)$ and $V(r)$ using Eqs. (12) and (23), evaluating the integrals using the trapezoid rule.

Theoretical and experimental values of $M(r), \sqrt{V(r)}$ and $\tau(r)$ at $r_{1}=0.4$ and $r_{2}=0.8$ are summarized in Table 1 , indicating that the experimental values match the theoretical predictions reasonably well. Our results also indicate that $M(r)$ and $\tau(r)$ are independent of $Q^{\prime}$ and therefore independent of whether we consider a pumping, injection or recovery process.

Table 1 about here ...

Table 2 shows $F(t \mid r)$ after times $t=M(r)$ and $t=\tau(r)$ in all experiments at both monitoring points. Our data indicate that after $t=M(r)$, approximately $55-67 \%$ of the process has taken place. In contrast, after $t=\tau(r)$, approximately $84-89 \%$ of the process has taken place. These results show that setting $m=1$ in Eq. (22) adequately estimates the amount of time required for the transient flow problem to effectively reach steady state conditions. We note that setting $m>1$ would lead to larger values of $\tau(r)$. However, the simplest possible choice of $m=1$ leads to a prediction of the time scale where about $87 \%$ of the transient response has taken place. Therefore, for practical purposes, setting $m=1$ leads to a useful and informative prediction of the relevant time at which the system effectively reaches the steady state.

Table 2 about here Fig. 3 shows the monitored transient head changes with the predicted theoretical values of $M(r)$ and $\tau(r)$ superimposed. Comparing the temporal head data and our estimates of $\tau(r)$ indicate that $\tau(r)$ is a useful estimate of the 
time required for the system to effectively reach steady state.

Fig. 3 here ...

\section{Summary and conclusions}

In this study we develop an analytical framework that can be used to: (1) estimate the time scale of the radial flow processes; (2) explicitly show how different hydraulic and geometric factors affect the time scales of the process; and (3) show how the time scales vary spatially within the flow domain. To achieve this we introduce two time scales: $M(r)$ and $\tau(r)$, using the concepts of mean action time (MAT) and variance of action time (VAT), respectively.

The key advantage of the MAT framework is that the mathematical expression for $M(r)$ explicitly shows how the geometry and hydraulic parameters affect the time scale of the flow process. In contrast, other definitions of the relevant time scale, such as using an arbitrary threshold, does not lead to mathematical expressions that are linked to hydraulic and geometric factors. Another limitation of using a threshold approach is that the time scale predicted itself would depend upon the choice of the threshold. In contrast, MAT avoids the need for defining an arbitrary, subjective threshold.

We also define the VAT, $V(r)$, and use this information to estimate the actual time needed to reach steady state after accounting for both the mean and the width of the PDF that governs the transition. Given $M(r)$ and $V(r)$ we define $\tau(r)=M(r)+m \sqrt{V(r)}$, as an estimate of the instant that explicitly accounts for the spread in the PDF. Here, $m$ is a positive integer, and to use this definition we must choose $m$. In this work we have used the simplest possible approach and have set $m=1$, meaning that $\tau(r)$ corresponds to the mean plus one standard deviation. Comparing our estimate of $\tau(r)$ with our laboratory 
scale data confirms that setting $m=1$ leads to a reasonable estimate of the time to reach steady state. For our test problem, $\tau(r)$ corresponds to the time required for approximately $84-89 \%$ of the transient response to have completed.

Our expressions for $M(r), V(r)$ and $\tau(r)$ explicitly relate the aquifer response time scale to various parameters including aquifer transmissivity, storage coefficient, well diameter and the location of the boundary. Our analysis shows that the groundwater response time scales are equivalent for pumping, injection and recovery processes, and are independent of the flow rate at the well. Moreover, our mathematical expressions quantify the spatial variations in the aquifer response time for radial flow processes including pumping, injection and recovery processes. These predictions are confirmed by our laboratoryscale measurements.

One of the limitations of our work is that we consider groundwater flow driven by a single pumping or injection well. A possible extension of our present study is to consider flow processes driven by multiple pumping and/or injection wells. Under these conditions we have to consider a more complicated two or three dimensional flow model in a Cartesian coordinate system. However, the general mathematical framework outlined here would still apply.

Acknowledgments: We thank the associate editor and two anonymous referees for taking a great deal of care in providing very detailed feedback on this work. This work was, in part, supported by a National Science Foundation Grant, NSF-EAR-0943679B. FJ was also supported by the Auburn University graduate student assistantship program. MJS was supported by the Australian Research Council (FT130100148). FJ, MJS and TPC developed the ideas for this work. FJ and MJS derived the mathematical model equations. FJ and TPC designed and completed the laboratory works. All three authors contributed to writing and approved the final version of the manuscript. Based 
375 on Clement's (2014) approach, the relative contributions are estimated to be:

376 FJ (60\%), MJS (20\%) and TPC (20\%). 
${ }_{404} r^{*}$ : characteristic length, [L];

$405 r_{w}^{\prime}$ : dimensional radius of the well, [L]; 
$406 r_{1}, r_{2}$ : dimensionless radial distance of monitoring points from the well, [-];

$407 r_{1}^{\prime}, r_{2}^{\prime}$ : dimensional radial distance of monitoring points from the well, [L];

${ }_{408} R^{\prime}$ : dimensional location of the boundary from the well center, [L];

$409 S$ : aquifer storage coefficient, [-];

${ }_{410} T^{\prime}$ : aquifer transmissivity, $\left[\mathrm{L}^{2} / \mathrm{T}\right]$;

$411 \quad t$ : dimensionless time, [-];

${ }_{412} t^{\prime}$ : dimensional time, $[\mathrm{T}]$;

$413 t^{*}$ : characteristic time, $[\mathrm{T}]$;

${ }_{414} t_{h}$ : hydraulic response time, [T]; (Gelhar and Wilson, 1974);

${ }_{415} V(r)$ : dimensionless variance of action time (VAT), [-];

${ }_{416} \alpha=r_{w}^{\prime} / R^{\prime},[-]$;

${ }_{417} \beta=\frac{Q^{\prime}}{2 \pi T^{\prime} h_{0}^{\prime} \alpha},[-]$;

${ }_{418} \delta$ : parameter indicating a specific time, $[\mathrm{T}]$;

${ }_{419} \tau(r)$ : dimensionless time scale $M(r)+m \sqrt{V(r)}$, [-];

${ }_{420} \phi(r)=g(r)\left[V(r)+T(r)^{2}\right],[-]$.

421 


\section{References}

[1] Bear, J., 1979. Hydraulics of groundwater. McGraw Hill, New York.

[2] Bredehoeft, J. D., Back, W., Hanshaw, B. B., 1982. Regional ground-water flow concepts in the United States: historical perspective. Geological Society of America Special Papers, 189, 295-316.

[3] Clement, T.P., 2014. Authorship matrix - a rational approach to quantify individual contributions and responsibilities in multi-author scientific articles. Science and Engineering Ethics. 20, 345361.

[4] Crank, J., 1975. The mathematics of diffusion. Oxford University Press, Oxford.

[5] Ellery, A. J., Simpson, M. J., McCue, S. W., Baker, R. E., 2012a. Moments of action provide insight into critical times for advection-diffusion-reaction processes. Physical Review E, 86(3), 031136.

[6] Ellery, A. J., Simpson, M. J., McCue, S. W., Baker, R. E., 2012b. Critical time scales for advection-diffusion-reaction processes. Physical Review E, 85(4), 041135.

[7] Erskine, A. D., Papaioannou, A., 1997. The use of aquifer response rate in the assessment of groundwater resources. Journal of Hydrology, 202(1), 373-391.

[8] Freeze, R. A., Witherspoon, P. A., 1966. Theoretical analysis of regional groundwater flow: 1. Analytical and numerical solutions to the mathematical model. Water Resources Research, 2(4), 641-656.

[9] Gelhar, L. W., Wilson, J. L., 1974. Ground-water quality modeling. Groundwater, 12(6), 399-408.

[10] Haitjema, H., 2006. The role of hand calculations in ground water flow modeling. Groundwater, 44(6), 786-791.

[11] Hantush, M. M., 2005. Modeling stream-aquifer interactions with linear response functions. Journal of Hydrology, 311(1), 59-79. 
[12] Jazaei, F., Simpson, M. J., Clement, T. P., 2014. An analytical framework for quantifying aquifer response time scales associated with transient boundary conditions. Journal of Hydrology, 519, 1642-1648.

[13] Kooi, H., Groen, J., Leijnse, A., 2000. Modes of seawater intrusion during transgressions. Water Resources Research, 36(12), 3581-3589.

[14] Manga, M.; 1999. On the timescales characterizing groundwater discharge at springs. Journal of Hydrology, 219(1), 56-69.

[15] McNabb, A., 1993. Mean action times, time lags, and mean first passage times for some diffusion problems. Mathematical and Computer Modelling 18, 123-129.

[16] McNabb, A., Wake, G.C., 1991. Heat conduction and finite measure for transition times between steady states. IMA Journal of Applied Mathematics 47, 193-206.

[17] Rousseau-Gueutin, P., Love, A. J., Vasseur, G., Robinson, N. I., Simmons, C. T., Marsily, G., 2013. Time to reach near-steady state in large aquifers. Water Resources Research, 49(10), 6893-6908.

[18] Schwartz, F. W., Sudicky, E. A., McLaren, R. G., Park, Y. J., Huber, M., Apted, M., 2010. Ambiguous hydraulic heads and 14C activities in transient regional flow. Groundwater, 48(3), 366-379.

[19] Simpson, M. J., Jazaei, F., Clement, T. P., 2013. How long does it take for aquifer recharge or aquifer discharge processes to reach steady state? Journal of Hydrology, 501, 241-248.

[20] Theis, C. V., 1940. The source of water derived from wells. Civil Engineering, 10(5), 277-280.

[21] Theis, C. V., 1935. The relation between the lowering of the piezometric surface and the rate and duration of discharge of a well using ground water storage. Washington, DC: US Department of the Interior, Geological Survey, Water Resources Division, Ground Water Branch.

[22] Townley, L. R., 1995. The response of aquifers to periodic forcing. Advances in Water Resources, 18(3), 125-146. 
476 [23] Li, W., Nowak, W., Cirpka, O. A., 2005. Geostatistical inverse modeling of 477 transient pumping tests using temporal moments of drawdown. Water Resources $478 \quad$ Research, 41(8), 1-13.

479 [24] Zhu, J., Yeh, T. C. J., 2006. Analysis of hydraulic tomography using temporal 480 moments of drawdown recovery data. Water Resources Research, 42(2), W02403. 


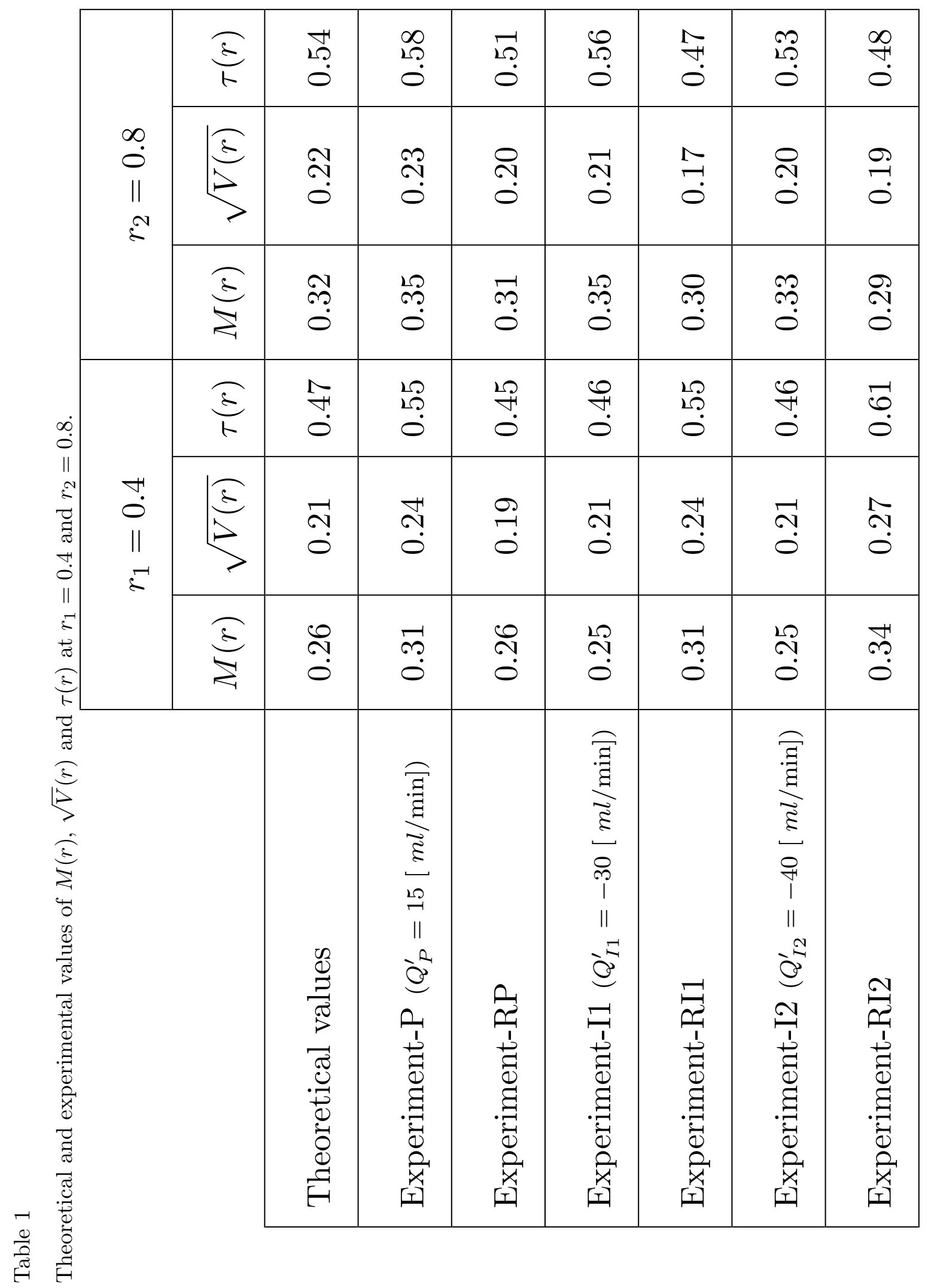




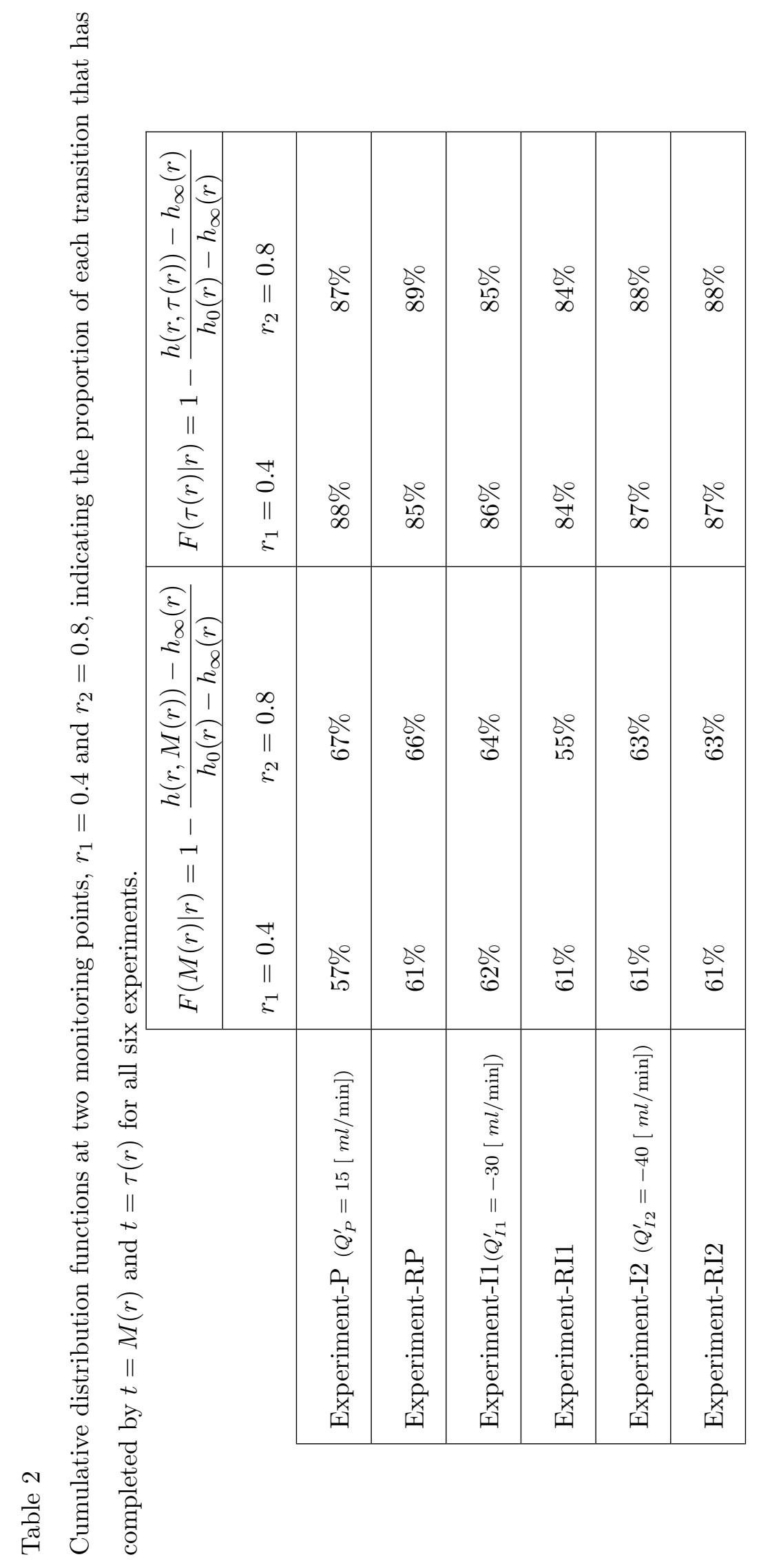




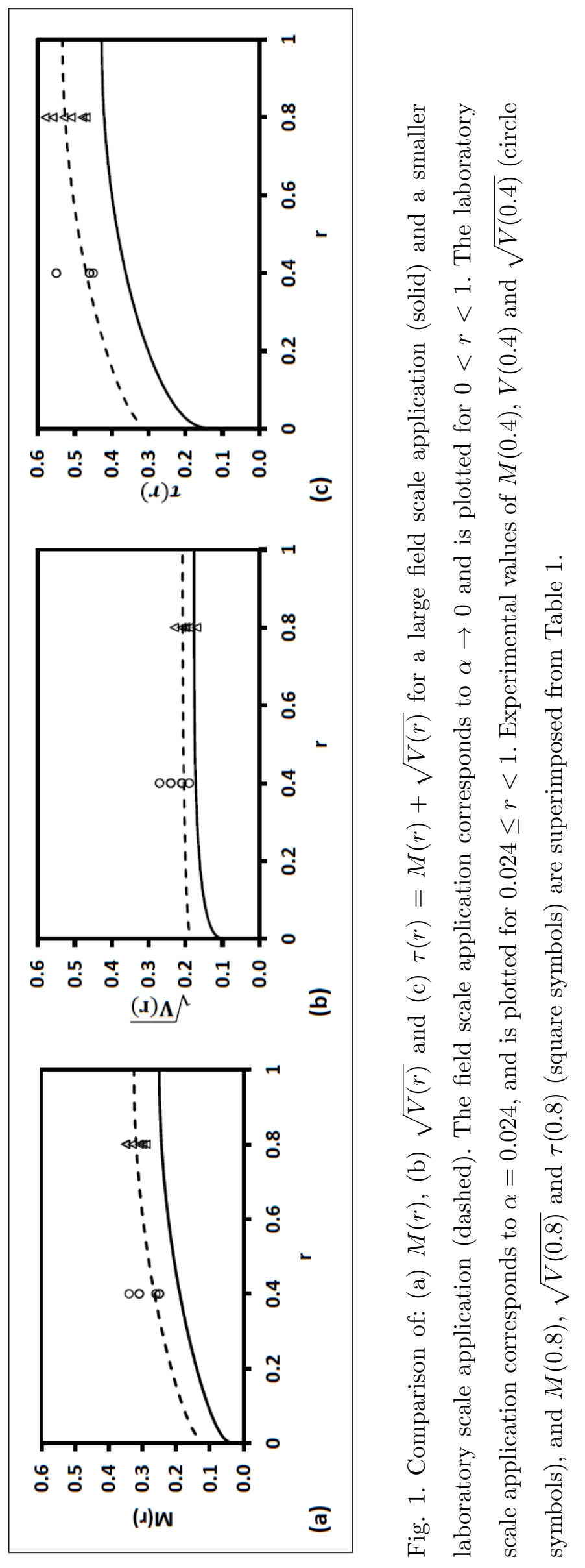




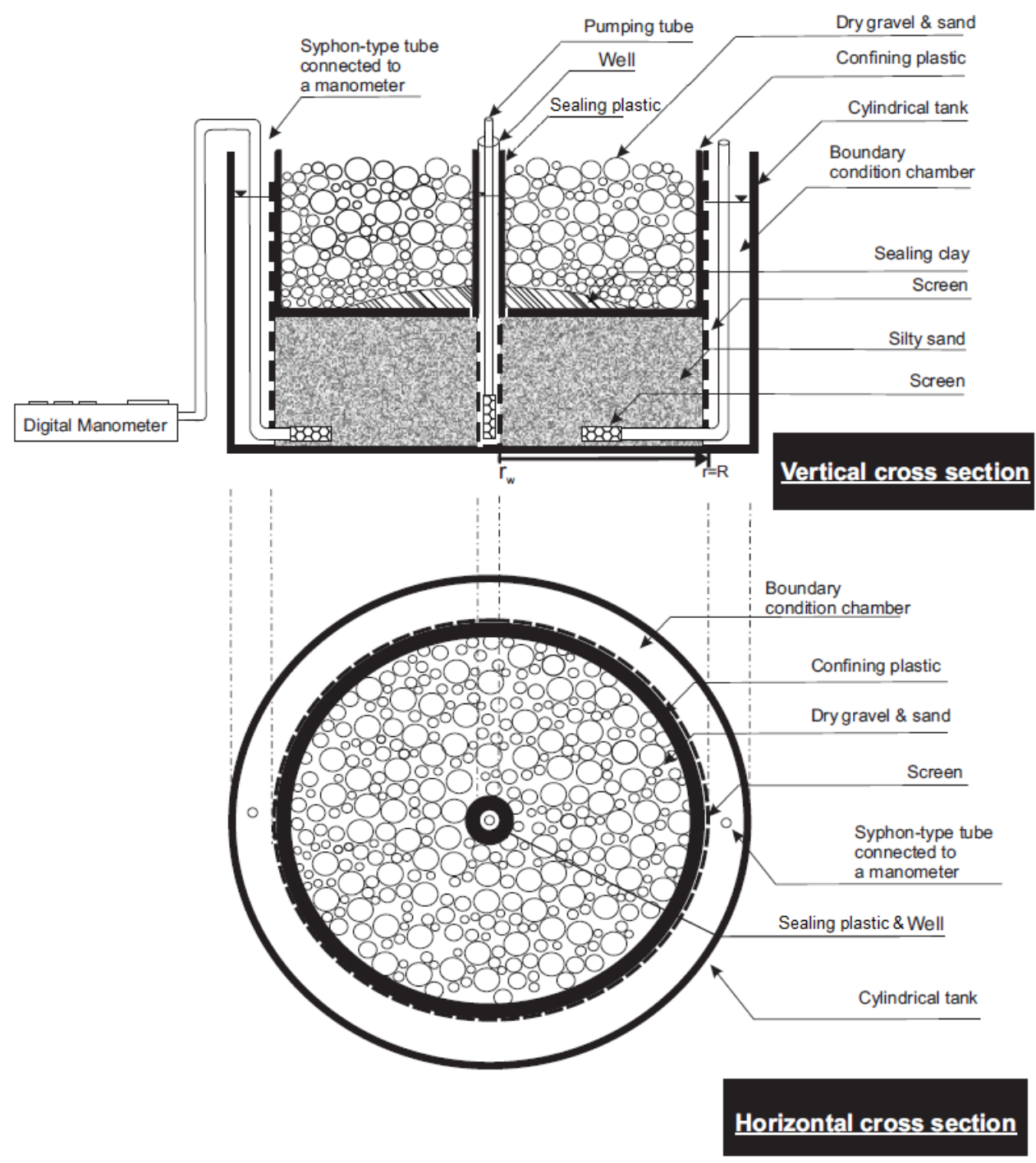

Fig. 2. Vertical and horizontal cross sections of the laboratory model. 


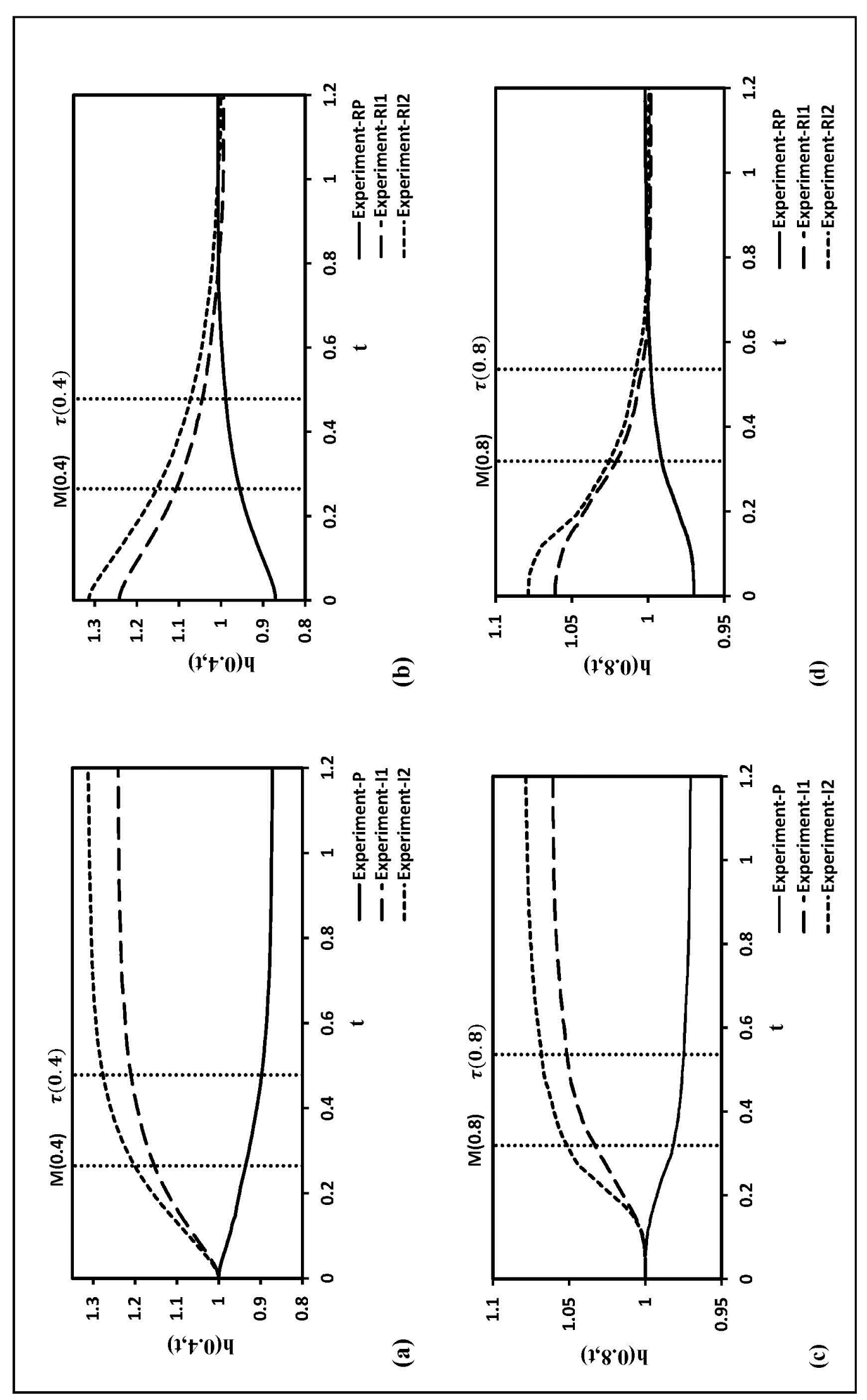

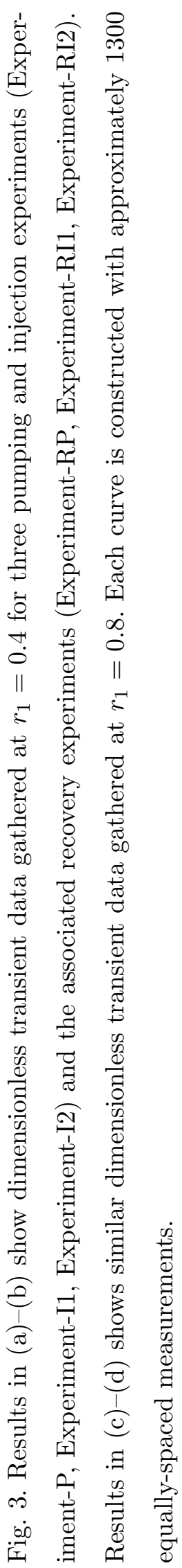

